\title{
Feasibility and safety of partial resection of the anterior insular cortex for microsurgical treatment of middle cerebral artery aneurysms located in the limen recess: patient series
}

\author{
Yosuke Akamatsu, MD, ${ }^{1,2}$ Hiroshi Kashimura, MD, ${ }^{1}$ Shunrou Fujiwara, $\mathrm{PhD},{ }^{2}$ Yoshitaka Kubo, $\mathrm{MD},{ }^{2}$ and Kuniaki Ogasawara, $\mathrm{MD}^{2}$ \\ ${ }^{1}$ Department of Neurosurgery, Iwate Prefectural Chubu Hospital, Kitakami, Japan; and ${ }^{2}$ Department of Neurosurgery, Iwate Medical University, Yahaba, Japan
}

\begin{abstract}
BACKGROUND When performing clip ligation of superior projecting aneurysms of the proximal (M1) segment of the middle cerebral artery (MCA), meticulous sylvian fissure dissection alone may be inadequate for safe clip application, especially in cases in which the aneurysm is buried in the limen recess, since the limen insulae may be positioned lateral to the aneurysm. In the present patient series, the authors present their surgical technique for clip ligation of aneurysms located in the limen recess, with partial resection of the limen insulae.

OBSERVATIONS A retrospective analysis of patients who had undergone clip ligation of MCA aneurysms located at the limen recess at a single institute was performed. Patients with angiographic and clinical follow-up data were considered eligible. A total of 11 aneurysms (4 ruptured and 7 unruptured aneurysms) in 11 patients were evaluated. Postoperative ischemic lesions were observed on images obtained within 1 week after surgery in $5(45.5 \%)$ patients who had undergone partial resection of the limen insulae, although none of them presented with neurological deterioration.

LESSONS Partial resection of limen insulae may be feasible to avoid severe ischemic complications following clip ligation of M1 aneurysms embedded in the limen recess.
\end{abstract}

https://thejns.org/doi/abs/10.3171/CASE21158

KEYWORDS middle cerebral artery aneurysms; M1 segment; limen recess; limen insulae; clipping

Superior projecting aneurysms of the proximal (M1) segment of the middle cerebral artery (MCA) usually arise at the origin of the lenticulostriate artery (LSA) or orbitofrontal artery. ${ }^{1-3}$ When performing clip ligation of these aneurysms, circumferential dissection of the aneurysm neck using a subfrontal operative view is the key to avoiding ischemic complications that may lead to severe motor and sensory deficits. ${ }^{3}$ Since superior projecting aneurysms of the M1 segment are often buried in the frontal lobe and adherent to the LSA or orbitofrontal artery, meticulous dissection of the sylvian and carotid cisterns is required to expose the aneurysms and surrounding structures without the need for extensive brain retraction. However, we sometimes experience difficulty in aneurysm exposure in patients with superior projecting M1 aneurysms in whom the aneurysm is buried in the limen recess, even after meticulous dissection of the sylvian fissure, since exposure of the aneurysm is restricted by the limen insulae. To date, few studies have focused on whether the location of the aneurysm relative to the surrounding brain structures affects achievement of an adequate surgical view when performing clip ligation of superior projecting M1 aneurysms, although prevention of injury to the LSA and orbitofrontal artery has often been discussed. ${ }^{3,4}$ In our clinical practice, we partially resect the limen insulae to expose aneurysms embedded in the limen recess in cases in which meticulous dissection of the sylvian fissure does not allow circumferential dissection of the aneurysm.

In this patient series, we present our experience with 11 patients with superiorly projecting M1 aneurysms located at the limen recess and discuss the feasibility and safety of our technique.

ABBREVIATIONS CTA = computed tomography angiography; DMCV = deep middle cerebral vein; DW-MRI = diffusion-weighted magnetic resonance imaging; ICG = indocyanine green; LSA = lenticulostriate artery; M1 segment = proximal segment; M2 segment = insular segment; MCA = middle cerebral artery; $\mathrm{mRS}=$ modified Rankin Scale; $\mathrm{SAH}=$ subarachnoid hemorrhage.

INCLUDE WHEN CITING Published June 7, 2021; DOI: 10.3171/CASE21158.

SUBMITTED March 18, 2021. ACCEPTED March 28, 2021.

(C) 2021 The authors, CC BY-NC-ND 4.0 (http://creativecommons.org/licenses/by-nc-nd/4.0/). 


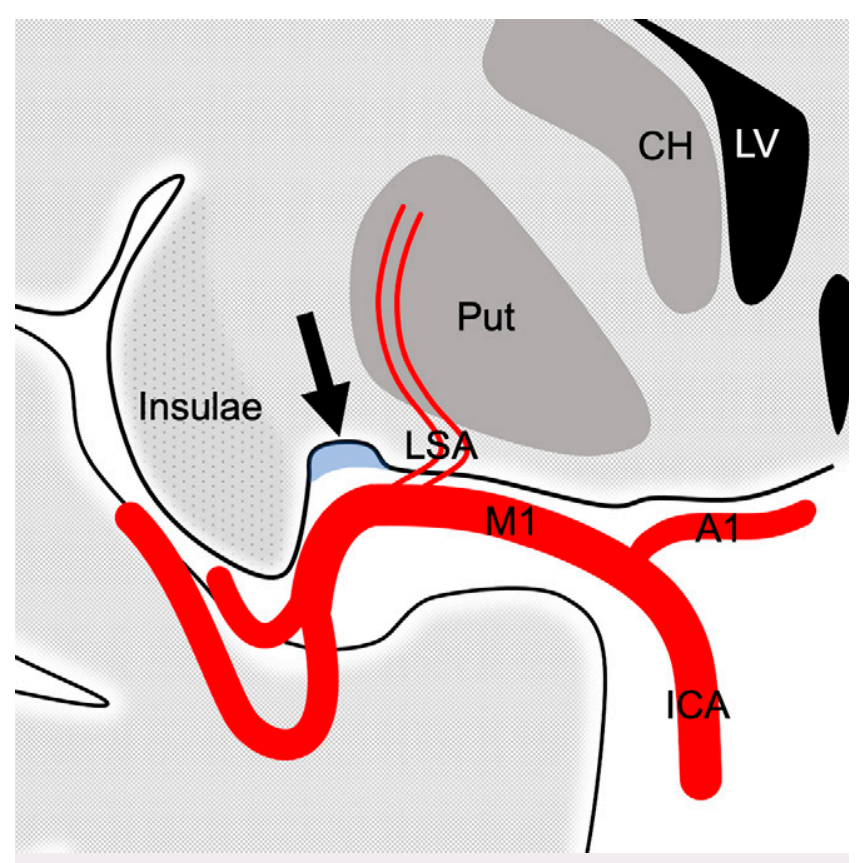

FIG. 1. Illustration of a coronal section at the level of the right anteroinferior insula showing the limen recess (light blue space, black arrow). $\mathrm{A} 1=$ proximal segment of the anterior cerebral artery; $\mathrm{CH}=$ caudate head; ICA = internal carotid artery; LV = lateral ventricle; Put = putamen.

\section{Study Description}

\section{Study Design, Setting, and Participants}

The clinical records of 219 consecutive patients with ruptured or unruptured MCA aneurysms (116 ruptured, 103 unruptured) who had undergone surgical clipping of the aneurysm at our hospital between May 2009 and April 2019 were retrospectively reviewed. From among these patients, those with MCA aneurysms located at the limen recess were included in the study. This study received institutional review board approval from the Iwate Prefectural Chubu Hospital prior to its commencement. Informed consent from the participants was obtained at the time of treatment.

\section{Variables and Data Source}

Medical records and angiographic images were reviewed for collection of data on patient demographics, clinical presentation, angiographic features of the aneurysm, and angiographic and clinical outcomes.

Patients with aneurysms located in the limen recess on coronal reformatted computed tomography angiography (CTA) images, which were obtained preoperatively, were included in the present study (Fig. 1). The location of the aneurysms was also verified intraoperatively. The features of the aneurysms were evaluated using preoperative three-dimensional CTA. Angiographic outcomes and the presence of ischemic complications were evaluated on CTA and magnetic resonance imaging studies obtained within 1 week after surgery by the treating neurosurgeon (H.K.) and an independent neurosurgeon who was not involved in the procedure (Y.A.). Clinical outcomes were evaluated during follow-up clinical visits. Any procedure-related complications, including any unanticipated adverse event, if present, were recorded. Postoperative morbidity was defined as permanent neurological deficits or changes in the modified Rankin Scale (mRS) score $\geq 1$ between pretreatment and posttreatment scores.

\section{Surgical Technique}

The patient is positioned supine. A curved linear scalp incision is made beginning at the zygomatic arch $1.5 \mathrm{~cm}$ anterior to the tragus and ending at the midline, just behind the hairline. A frontotemporal craniotomy is performed with flattening of the lesser wing of the sphenoidal bone until the lateral edge of the superior orbital fissure is reached. The dura is opened with a semicircular incision. The superficial sylvian, carotid, and lamina terminalis cisterns are sharply dissected, and the M1 and insular (M2) segments are exposed. Then, in order to achieve sufficient visualization of the aneurysm neck and critical branches, dissection of the aneurysm and

TABLE 1. Summary of the 11 cases with superior projecting MCA aneurysms located at the limen recess and procedure-related outcomes

\begin{tabular}{|c|c|c|c|c|c|c|c|c|c|c|}
\hline $\begin{array}{l}\text { Case } \\
\text { No. }\end{array}$ & $\begin{array}{l}\text { Age (yrs), } \\
\text { Sex }\end{array}$ & Presentation & $\begin{array}{l}\text { Related } \\
\text { Arteries }\end{array}$ & $\begin{array}{l}\text { Lesion } \\
\text { Size }(\mathrm{mm})\end{array}$ & $\begin{array}{c}\text { Partial } \\
\text { Resection } \\
\text { of Insular Cortex }\end{array}$ & $\begin{array}{c}\text { Total TO } \\
\text { Time (mins) }\end{array}$ & $\begin{array}{l}\text { Postop DW- } \\
\text { MRI Lesions }\end{array}$ & \multicolumn{2}{|c|}{ mRS Score } & $\begin{array}{c}\text { Newly } \\
\text { Developed } \\
\text { PND }\end{array}$ \\
\hline 2 & $64, F$ & Incidental & OFA & 5.2 & No & NA & IC, frontal cortex & 0 & 4 & Yes \\
\hline 3 & $76, F$ & Incidental & OFA, LSA & 4.6 & Yes & 13.4 & NA & 0 & 0 & No \\
\hline 6 & $66, F$ & Incidental & OFA & 5.5 & Yes & 15.48 & NA & 0 & 0 & No \\
\hline 7 & $57, M$ & Incidental & M1-M2 & 7.0 & Yes & 17.52 & Insula & 0 & 0 & No \\
\hline 8 & $71, \mathrm{M}$ & SAH (H \& K: IV) & M1-M2 & 11.1 & Yes & 5.0 & Insula & 5 & 4 & No \\
\hline 9 & $49, \mathrm{~F}$ & SAH (H \& K: II) & OFA & 5.0 & Yes & 11.0 & NA & 3 & 0 & No \\
\hline 10 & $87, \mathrm{~F}$ & SAH (H \& K: IV) & OFA, LSA & 3.4 & Yes & 3.77 & Insula & 5 & 5 & No \\
\hline
\end{tabular}

$\mathrm{H} \& \mathrm{~K}=$ Hunt and Kosnik grade of SAH; IC = internal capsule; NA = not available; OFA = orbitofrontal artery; PND = postoperative neurological deficit; TO = temporary occlusion. 
surrounding structures is attempted by careful release of the arachnoid membrane under light dynamic retraction of the insular cortex. If the aneurysm is hidden by the limen insulae even after the procedure mentioned above, the aneurysm neck is exposed following pial incision of the anteroinferior part of the insular cortex up to about $5 \mathrm{~mm}$ in width by resecting the cortex with a sucker (2-mm width). In cases in which the LSA or deep middle cerebral vein (DMCV) is found on the insular cortex, the cortical incision is limited to the area of the cortex where these vessels are not overlying. Using this surgical corridor, the aneurysm and surrounding structures, such as the LSA and orbitofrontal artery, can be circumferentially dissected.
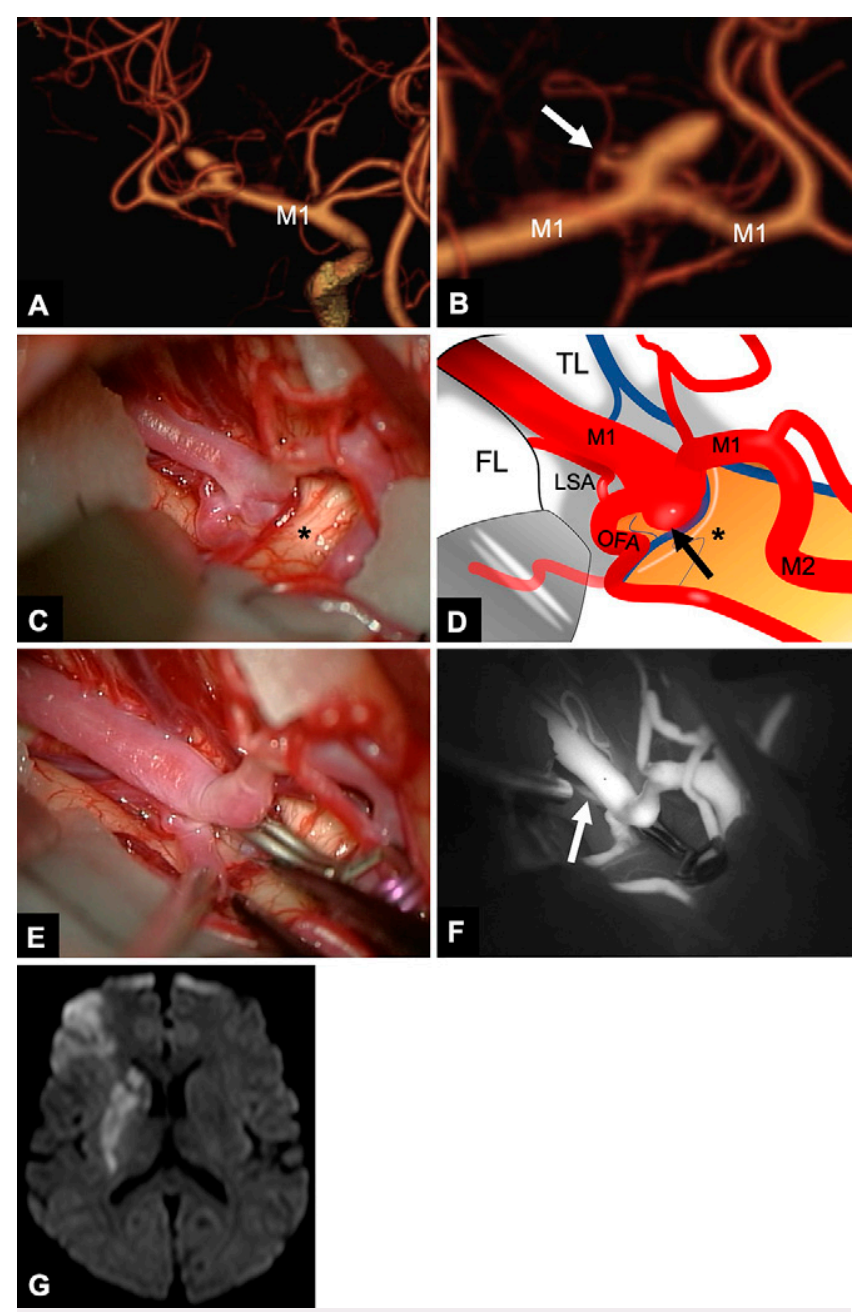

FIG. 2. Anteroposterior (A) and posteroanterior (B) views of three-dimensional CTA in case 2 demonstrating the superior projecting M1 aneurysm arising from the origin of the orbitofrontal artery (white arrow). Intraoperative photograph demonstrating the aneurysm embedded in the limen recess (asterisk) (C) and an illustration depicting the structures surrounding the aneurysm (black arrow) (D). Note how exposure of the aneurysm was restricted by the limen insulae (asterisk) (D). Intraoperative photograph (E) following clip ligation of the aneurysm without partial resection of the limen insulae. ICG angiography of the same surgical corridor demonstrating patency of the orbitofrontal artery and LSA (white arrow) (F). DW-MRI scan obtained postoperatively, revealing ischemic lesions in the territories of the orbitofrontal artery and the LSA

(G). $\mathrm{FL}=$ frontal lobe; OFA = orbitofrontal artery; $\mathrm{TL}=$ temporal lobe.
Then, a single or multiple titanium clips are applied to obliterate the aneurysm without interfering with blood flow in the afferent and efferent arteries. Obliteration of the aneurysm and patency of the surrounding vessels are confirmed intraoperatively by Doppler flowmetry and indocyanine green (ICG) video angiography.

\section{Results}

A total of $11(5.0 \%)$ aneurysms at the limen recess were identified from among $219 \mathrm{MCA}$ aneurysms treated with microsurgical clipping (in 3 men and 8 women). The median age at treatment was 66 years (range 35-87 years). There were 4 ruptured and 7 unruptured aneurysms. Among the cases with aneurysmal rupture, 2 patients presented with Hunt and Kosnik grade II subarachnoid hemorrhage (SAH), and the other two had grade IV SAH. The orbitofrontal artery was the most commonly involved branch artery $(54.5 \%)$, followed by early bifurcation of M1-M2 (27.2\%) and the LSA (18.2\%). The median size of the aneurysms was $5.1 \mathrm{~mm}$ (range $3.0-11.1 \mathrm{~mm}$ ).

Clip ligation of the aneurysms was performed following partial resection of the anterior insular cortex in 10 of the 11 cases. In all cases, the aneurysms were embedded in the limen recess even after meticulous dissection of the sylvian fissure. During the procedure, temporary occlusion of the M1 segment was performed in 8 of the 11 patients $(72.7 \%)$. The median value of the total temporary occlusion time of the M1 segment was 10.78 minutes (range 3.77-17.52 minutes). In each case, the duration of every temporary clipping was limited to up to 5 minutes. Postoperative diffusion-weighted magnetic resonance imaging (DW-MRI) performed within 1 week after surgery revealed ischemic lesions in 5 patients (45.5\%). Among them, none of the patients who had undergone partial resection of the limen insulae presented with neurological deterioration despite high-intensity lesions at the insular cortex. However, 1 patient without partial resection of the limen insulae presented with symptomatic cerebral infarction in the internal capsule and prefrontal cortex due to injury to the LSA and orbitofrontal artery. The baseline characteristics of the enrolled patients and the procedure-related outcomes are shown in Table 1.

\section{Illustrative Cases}

Case 2

A 64-year-old woman was referred to our hospital for treatment of an unruptured right MCA aneurysm. Three-dimensional CTA showed a superior projecting aneurysm arising at the origin of orbitofrontal artery (Fig. $2 \mathrm{~A}$ and $\mathrm{B}$ ), for which a right frontotemporal

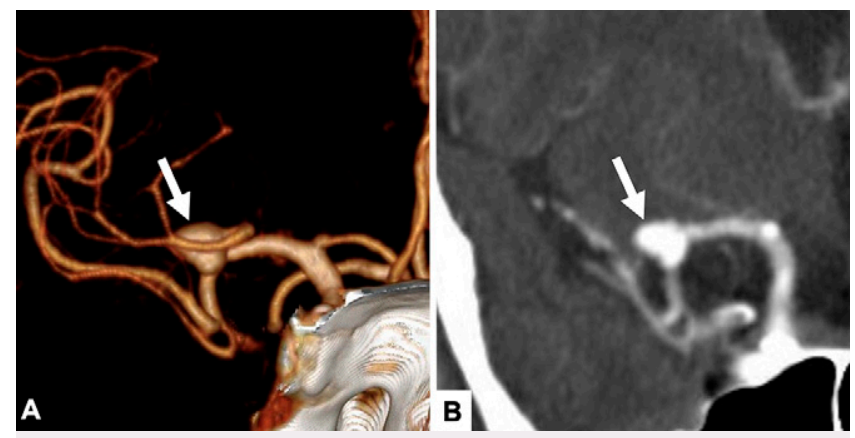

FIG. 3. Anteroposterior $(\mathbf{A})$ view of a reconstructed three-dimensional CTA image and a coronal source image (B) in case 7 demonstrating the superior projecting M1 aneurysm embedded in the limen recess (white arrows). 
craniotomy was performed. Despite meticulous dissection of the sylvian fissure and the M1 and M2 segments, about three-quarters of the aneurysm dome was embedded in the limen insulae (Fig. $2 \mathrm{C}$ and $\mathrm{D}$ ). The aneurysm clip was applied at the aneurysm neck through the minimal space obtained without partial resection (Yasargil titanium mini clips, no. FT727T, Aesculap AG \& Co.) (Fig. 2E). Although intraoperative ICG angiography revealed patency of the orbitofrontal artery (Fig. 2F), the patient developed left hemiparesis postoperatively. DW-MRI performed 1 day after surgery demonstrated cerebral infarction in the territories of the orbitofrontal artery and the LSA, probably due to flow impairment in the LSA resulting from kinking of the orbitofrontal artery (Fig. 2G). Although the patient's symptoms gradually improved, mild hemiparesis persisted 6 months after surgery (mRS score 4).

\section{Case 7}

A 57-year-old man was referred to our hospital for treatment of an incidentally diagnosed unruptured right MCA aneurysm. Threedimensional CTA showed a superolaterally projecting aneurysm arising at the origin of the orbitofrontal artery (Fig. $3 \mathrm{~A}$ ). A coronal (Fig. 3B) source image on CTA demonstrated that the aneurysm was located just medial to the limen insulae, indicating that it was embedded in the limen recess. A right frontotemporal craniotomy was performed, and the sylvian fissure was opened widely. Since we could not achieve adequate exposure of the aneurysm (Fig. 4A and $B$ ), partial resection of the limen insulae was performed. During this procedure, a small anterior insular vein was sacrificed. By gently retracting the aneurysm dome, the position of the LSA could be clearly appreciated (Fig. 4C and D). The aneurysm was subsequently obliterated using an interlocking, tandem clipping technique under temporary occlusion of M1 (Yasargil titanium standard clips, no. FT623T and FT597T) (Fig. 4E), and complete obliteration of the aneurysm was confirmed using intraoperative ICG angiography (Fig. 4F). During the entire procedure of exposing the aneurysm and performing the clipping procedure, temporary clipping of the artery proximal to the lesion was required 5 times (total time 17.52 minutes). Postoperative three-dimensional CTA revealed patency of the orbitofrontal artery originating from the M1 segment, as well as obliteration of the aneurysm. Although postoperative DW-MRI revealed a minimal high-intensity lesion in the insula (Fig. 4G), the patient was discharged without any neurological deficits.

\section{Discussion \\ Observations}

Despite the present endovascular era, microsurgical clip ligation remains the mainstay for MCA aneurysms because of their specific morphological characteristics, such as a wide neck and asymmetrical branches that are incorporated into the aneurysm neck. ${ }^{5-8}$ When performing clip ligation, superiorly projecting aneurysms arising from the M1 segment represent one of the most complicated aneurysms, requiring dissection of the LSA from the aneurysm to avoid postoperative morbidities. ${ }^{1,4}$ Although most of these aneurysms can be exposed by meticulous dissection of the sylvian fissure, some of the aneurysms cannot be sufficiently dissected if they are encased in a sulcus-like structure (mean width $15 \mathrm{~mm}$ ), referred to as the "limen recess," between the medial border of the limen insulae and the lateral limit of the anterior perforating substance. ${ }^{9}$

In the present series, lateral and posterior dissection of the aneurysms via the subfrontal operative corridor was limited in all
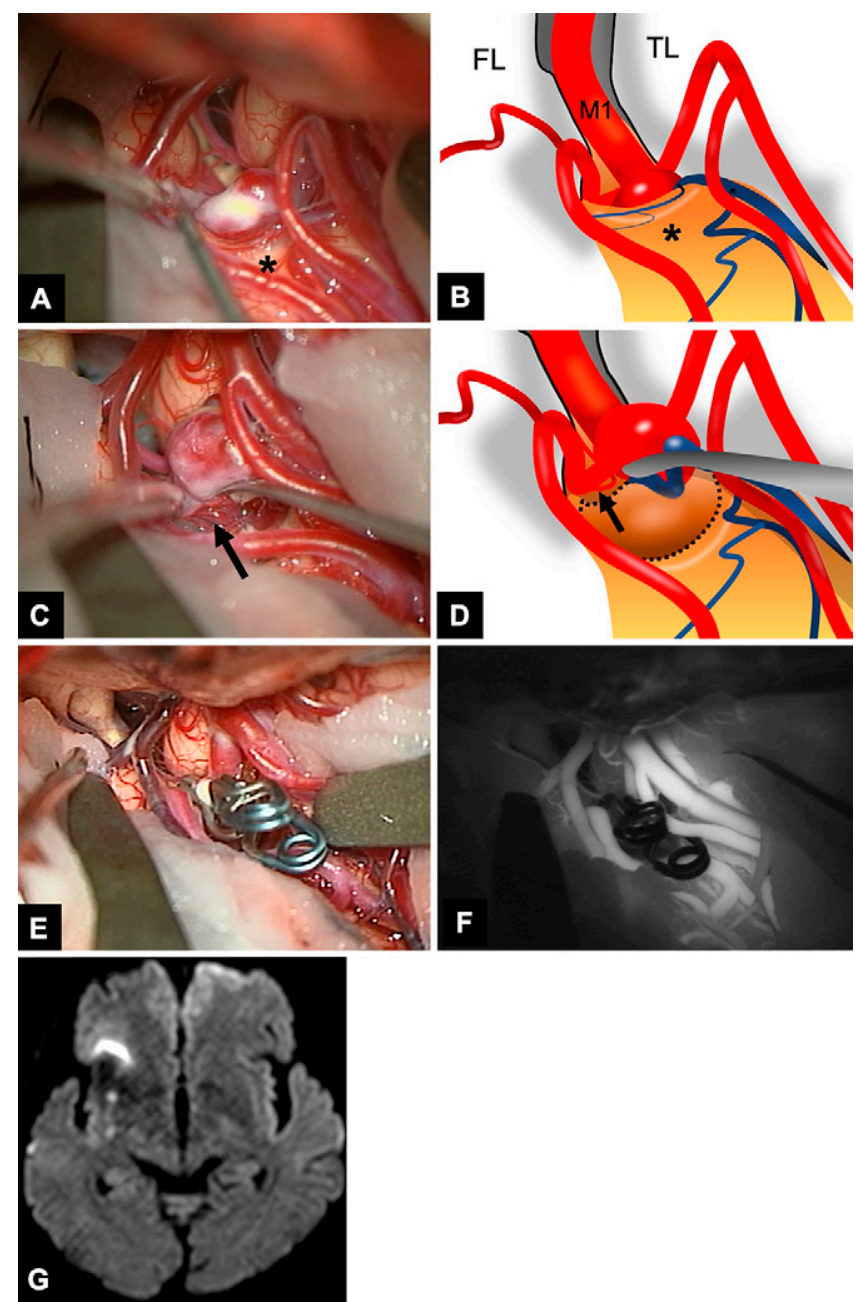

FIG. 4. Initial exposure following wide opening of the sylvian fissure in case 7 demonstrating the aneurysm covered by the limen insulae (asterisks) and embedded in the limen recess. A: Intraoperative photograph. B: Illustration. Circumferential exposure of the aneurysm following partial resection of the limen insulae, allowing clear exposure of LSA (black arrow in C). C: Intraoperative photograph. D: Illustration. Note the dissection plane of the limen insular (black dotted line in D) and the origin of the lenticulostriate artery (arrow) in the illustration. Intraoperative photograph (E) and ICG angiography image (F) after clip ligation of the aneurysm confirming complete obliteration of the aneurysm. DW-MRI scan obtained postoperatively, revealing ischemic lesions in the right insular area, despite the patient being asymptomatic $(\mathbf{G})$.

cases despite meticulous dissection of the sylvian fissure. Following our experience of a patient who presented with complications related to occlusion of the LSA and the orbitofrontal artery (case 2), we have been performing clip ligation of these aneurysms, with partial resection of the insular cortex covering the lateral and posterior walls of the aneurysm to achieve circumferential dissection of the aneurysms. Thereafter, we have not observed postoperative neurological deterioration in any of the 10 patients with $\mathrm{M} 1$ aneurysms in whom this procedure has been performed.

There are general concerns regarding whether partial resection of the limen insulae can cause morbidity as a result of direct injury to the insular cortex, the LSA, and the deep-seated cortical veins. 
In terms of the influence of direct injury to the anterior insular cortex, Sanai et al. ${ }^{10}$ reported that the anterior zone of the insulae is amenable to a greater extent of resection without postoperative morbidity, suggesting that this procedure can be performed relatively safely. Therefore, if circumferential dissection of aneurysms in the limen recess is restricted by the insular cortex, partial resection of the limen insulae may be less harmful, unless the medial resection plane extends beyond the limen recess. Consistent with this supposition, neurological deterioration did not occur following partial resection of the limen insulae in our series, although small ischemic lesions developed in the anterior part of some of the insular cortices.

Tanriover et al. ${ }^{9}$ reported a thorough cadaveric study in which they demonstrated that the limen recess lacks important perforating arteries penetrating the anterior perforated substance. Therefore, partial resection of the insular cortex does not necessarily injure the LSA, unless the resection extends medially to the limen recess. This anatomical feature of the limen recess would explain why none of our patients presented with neurological deterioration even after partial resection of the limen insulae.

The DMCV is the largest and most consistent branch of the basal vein of Rosenthal, which is formed by the union of insular veins, including the anterior, precentral, central, and posterior insular veins, near the limen insulae. ${ }^{11-14}$ Thus, this vein can be an obstacle during partial resection of the limen insulae or clip application for aneurysms at the limen recess. In the present series, the main trunk of the DMCV was preserved in all cases, although some small anterior insular veins were sacrificed. However, we did not experience complications related to venous congestion, hemorrhage, or edema caused by sacrifice of the anterior insular veins.

\section{Lessons}

Some superior projecting aneurysms in the M1 segment tend to be embedded in the limen recess. When performing clip ligation of these aneurysms, since the aneurysms and surrounding structures, such as the LSA, are obstructed by the limen insulae, dissection of the sylvian fissure alone is insufficient to secure a safe working corridor for clip ligation. In such cases, partial resection of the limen insulae could be feasible for microsurgical clipping of aneurysms embedded in the limen recess.

The current study is limited by the fact that it was a retrospective review of a small case series from a single institution. Given the patient series size and limited outcome data, larger numbers of patients will be needed to confirm the safety and feasibility of the present technique and to determine when its use is most appropriate.

\section{References}

1. Hosoda K, Fujita S, Kawaguchi T, et al. Saccular aneurysms of the proximal (M1) segment of the middle cerebral artery. Neurosurgery. 1995;36(3):441-446.

2. Pia HW. Aneurysms of the middle cerebral artery. In: Pia HW, Langmaid C, Zierski J, eds. Cerebral Aneurysms: Advances in Diagnosis and Therapy. Springer; 1979:116-118.
3. Yasargil MG. Middle cerebral artery aneurysms. In: Yasargil MG, ed. Microneurosurgery. Vol 2. Georg Thieme Verlag; 1984:124-164.

4. Iwama T, Yoshimura S, Kaku Y, Sakai N. Considerations in the surgical treatment of superior-wall type aneurysm at the proximal (M1) segment of the middle cerebral artery. Acta Neurochir (Wien). 2004;146(9):967-972.

5. Dashti R, Hernesniemi J, Niemelä M, et al. Microneurosurgical management of middle cerebral artery bifurcation aneurysms. Surg Neurol. 2007;67(5):441-456.

6. Jeon HJ, Kim SY, Park KY, et al. Ideal clipping methods for unruptured middle cerebral artery bifurcation aneurysms based on aneurysmal neck classification. Neurosurg Rev. 2016;39(2):215-224.

7. Lawton M. Middle cerebral artery aneurysms. In: Connerly K, Wachinger M, Chernow B, et al., eds. Seven Aneurysms: Tenets and Techniques for Clipping. Thieme; 2011:65-93.

8. Washington CW, Ju T, Zipfel GJ, Dacey RG Jr. Middle cerebral artery bifurcation aneurysms: an anatomic classification scheme for planning optimal surgical strategies. Neurosurgery. 2014; 10(suppl 1):145-155.

9. Tanriover N, Rhoton AL Jr, Kawashima M, et al. Microsurgical anatomy of the insula and the sylvian fissure. J Neurosurg. 2004;100(5): 891-922.

10. Sanai N, Polley MY, Berger MS. Insular glioma resection: assessment of patient morbidity, survival, and tumor progression. J Neurosurg. 2010;112(1):1-9.

11. Huang YP, Wolf $B S$. The basal cerebral vein and its tributaries. In: Newton TH, Potts DG, eds. Radiology of the Skull and Brain. Vol 2. CV Mosby; 1974:2111-2154.

12. Ono M, Rhoton AL Jr, Peace D, Rodriguez RJ. Microsurgical anatomy of the deep venous system of the brain. Neurosurgery. 1984;15(5):621-657.

13. Serrats AA, Vlahovitch $B$, Parker $S A$. The arteriographic pattern of the insula: its normal appearance and variations in cases of tumour of the cerebral hemispheres. J Neurol Neurosurg Psychiatry. 1968;31(4):379-390.

14. Wolf BS, Huang YP. The insula and deep middle cerebral venous drainage system: normal anatomy and angiography. Am J Roentgenol Radium Ther Nucl Med. 1963;90:472-489.

\section{Disclosures}

The authors report no conflict of interest concerning the materials or methods used in this study or the findings specified in this paper.

\section{Author Contributions}

Conception and design: Kashimura, Akamatsu. Acquisition of data: Kashimura, Akamatsu, Kubo. Analysis and interpretation of data: Kashimura, Akamatsu, Kubo. Drafting the article: Akamatsu, Ogasawara. Critically revising the article: Akamatsu, Ogasawara. Reviewed submitted version of manuscript: Akamatsu, Fujiwara, Ogasawara. Approved the final version of the manuscript on behalf of all authors: Kashimura. Administrative/technical/material support: Ogasawara. Study supervision: Ogasawara.

\section{Correspondence}

Hiroshi Kashimura: Iwate Prefectural Chubu Hospital, Iwate, Japan. hkashimura@icloud.com. 